\title{
Clinical Trial Eligibility Criteria
}

National Cancer Institute

\section{Source}

National Cancer Institute. Clinical Trial Eligibility Criteria. NCI Thesaurus. Code C16112.

Characteristics which are necessary to allow a subject to participate in a clinical study, as outlined in the study protocol. The concept covers inclusion and exclusion criteria. 